IRSTI 27.35.51

\title{
Temperature-dependent quantum pair potentials and ionization in Helium-like plasmas
}

\author{
H. Rahal, C. Deutsch* and M.M. Gombert \\ Laboratory on physics of gases and plasmas, Université Paris XI, 91405 Orsay, France \\ *e-mail: claude.deutcsch@u-psud.fr
}

\begin{abstract}
Diffraction corrected effective and Coulombic electron-ion interaction are worked out for helium like ions in plasmas of warm dense matter (WDM) and chemically-reacting concern, through explicit wave functions derived within an appropriate quantum defect framework. Very significant departures from the usual and corresponding hydrogenlike expressions are then witnessed. To do so, we strongly rely on a coherent quantum defect model (QDM) making use of parameters qualifying departures from hydrogenic bound states with same quantum numbers for negative energies as well as phase shift corrections for positive energy levels. Such an approach is also completed with a very accurate Hartree-Fock (HF) approximation for the ground state. The QDM then approaches the Slater sum-over-state- for the binary electron-ion where the pertaining ion retains an electron on its $1 \mathrm{~s}$ hydrogenlike ground state. The resulting electron-ion effective interactions are then analytically derived through classical pair correlation functions. These latter are thoroughly contrasted to their hydrogenlike homologues. In addition,ionization in a 3-component electron-two ion system is also given a certain attention.
\end{abstract}

Keywords: Helium-like ions, diffraction pseudopotentials, warm dense matter, ionization.

PACS numbers: 52.27.Cm,52.70.-m; 31.10.+z; 52.25.Jm.

\section{Introduction}

To deal efficiently with basic and statistical physics concepts of central concern in dense and partially ionized plasmas, one is often confronted to the pitfall arising from the short range divergence of the ubiquitous electron-ion attraction [1-9]. A straightforward and pertinent approach allowing to circumvent this difficulty while maintaining a quasiclassical setting for equilibrium and transport properties is the mitigation at high temperature ( $T \geq 1$ Ry for low $\mathrm{Z}$ ions) of the given attraction through diffraction corrections due to the uncertainty principle. Up to now this standard procedure remains restricted to hydrogen-like electron ion interactions. This situation is mostly due to the identification of Gibbs potential with corresponding Slater sum requiring explicit wave function for the given pair of unlike charges. Moreover, when one is confronted to heavy ions $(Z>>1)$ retaining many bound planetary electrons, a Thomas-Fermi like description [10] is then usually in order, thus avoiding the use of explicit wave functions. However, strong and persistent request, mostly from the warm dense matter (WDM) community [11-12] stressing strongly coupled plasmas with solid density and $\mathrm{T}(\mathrm{eV}) \leq 10$, as well as accurate diagnostics of ion-plasma interaction $[13,14]$, put a recent and urgent emphasis on Helium like ions, i.e. with nuclei (any Z) retaining a strongly bound ls electron.

Accurate analysis of equilibrium and transport properties of helium-like chemically reacting plasmas [15] also features a significant domain of present concern where He-like Coulomb-like interactions are also urgently requested. In order to appreciate the present Slater sum approach within the perspective afforded by the present very active field of simulation techniques dedicated to WDM investigations based on path integral Monte-Carlo (PIMC) methods or on the extraction of ion-ion effective interactions [16-19] from N-body quasiclassical approaches, one witnesses an urgent demand for accurate non hydrogenic electron-ion pseudopotentials. In particular, the matching of PIMC thermodynamics at high temperature with the 
Debye one [16] requires that partial ionization and ion excitation be carefully accounted for temperature higher than the helium-like ion ionization energy (Eq.(27) in the sequel).

In this report, we intend to demonstrate an analytic derivation of the pertaining electron- ion pseudopotential, for this specific 3-body problem. Toward this goal we systematically rely on a consistent quantum defect model (QDM) [20, 32] based on parameters $a_{b}$ qualifying departures from hydrogenic bound states energies and $a_{c}$ for the phase shifts of the positive energy levels. Such an approach, completed with a HF ground state description [23] is validated by its overwhelming success in reproducing helium atom properties as quantitatively accurate as the hydrogen atom ones.

The present work is thus structured as follows. Sec. 2 develops the quantum defect model (QDM) approach to the Slater Sum-over- states for the binary system electron-ion $Z_{1}\left(Z_{1}=Z-1\right)$, with ion $Z_{1}$ kept on its hydrogenic $1 \mathrm{~s}$ ground state. Concommittent spin effects are also paid a due attention. The given temperature-dependent e-ion $Z_{1}$ effective interactions are then analytically detailed in Sec. 3, through the corresponding pair correlation function. The novel helium-like pseudopotentials are systematically contrasted to their hydrogen-like homologues, taken in a sequence with increasing precision. Ionization ion in a 3-component e-ion Zion $Z_{1}$ dense plasma is worked out in Sec. 4, with the aid of canonical free energy including electron exchange as well as quantum corrections featuring QDM parameters for e-ion $Z_{1}$ excited states. The following analysis implies that the plasma electrons remain classical (non degenerate), thus keeping their Debye length larger than the de-Broglie thermal one and still smaller than their average interdistance.

\section{Slater sum approach (SSA)}

\subsection{Bound state contributions}

Our basic concern is the plasma electron-ion effective interaction (pseudo-potentiel) in a He-like plasma where most of the ions (nonrelativistic) have the charge $Z_{1}=Z-1$, i.e. retain a strongly $1 \mathrm{~s}$ bound orbital electron. As in the well-known H-like the searched pseudopotential writes as $\left(\beta=1 / \mathrm{k}_{\beta} \mathrm{T}\right)$

$$
\mathrm{U}(\mathrm{r})=-\frac{1}{\beta} \ln [\mathrm{g}(\mathrm{r})]
$$

in terms of the $\mathrm{e}-\mathrm{Z}_{1}$ ion canonical correlation function. The main focus of the present work is to demonstrate a complete calculation of $\mathrm{g}(\mathrm{r})$ based on the present knowledge of the $\mathrm{He}$ atom physics. Numerically speaking, the latter is presently as accurate as the $\mathrm{H}$ atom one [20]. In order to make our derivation of present $\mathrm{e}-\mathrm{Z}_{1}$ effective interaction as close as possible as to its Hydrogen like homologue with ion of charge $\mathrm{Z}$, we shall elaborate our further developments on the quantum defect method [20]. Such an approach should secure an immediate matching with hydrogen-like (non relativistic) wave functions of the present QDM ones. Then, the corresponding 2-body and bound state part of $\mathrm{g}(\mathrm{r})$ appears under the H-like form $\left(\beta=\left(1 / \mathrm{k}_{\beta} \mathrm{T}\right)\right.$

$$
\begin{aligned}
g_{L}(r)= & \frac{1}{4 \pi}\left(\frac{2 \pi \beta \hbar^{2}}{m}\right)^{3 / 2} \sum_{n=1}^{\infty} \sum_{\ell=0}^{n-1}(2 \ell+1) \times \\
& \times \exp \left(-\beta E_{n l}\right)\left|R_{n \ell}(r)\right|^{2}
\end{aligned}
$$

in terms of $(n, \ell) H$-like excited states ( $m$ is the electron mass) with energy $E_{n l}$ and wave-function $\mathrm{R}_{\mathrm{nl}}(\mathrm{r})$ derived from their $\mathrm{H}$-homologues though the QDM procedure detailed below. The first term $\mathrm{n}=$ $1, \ell=0$ pertaining to ground state is given a specific Hartree-Fock attention with the parametrized expression,

$$
\frac{1}{2 Z_{1}^{3 / 2}} \mathrm{R}_{\mathrm{F}}(\mathrm{r})=\sum_{\mathrm{i}=0}^{2} \mathrm{c}_{\mathrm{i}}\left(\frac{\mathrm{k}_{\mathrm{i}}}{\mathrm{Z}_{1}}\right)^{3 / 2} \exp \left(-\mathrm{k}_{\mathrm{i}} \mathrm{r}\right)
$$

by Clementi and Roetti [23]. Some excerpts of the required parameters tabulations are given on Table 1.

The corresponding $\mathrm{g}_{\mathrm{L}}(\mathrm{r})$ contribution is adequately parametrized with $\xi=\frac{L}{\lambda}=Z_{1} \beta^{1 / 2}$, ratio of classical Landau length to the De Broglie electron wavelength. So, with $x=\frac{r}{\lambda}$, the HF partial correlation featuring the fundamental He-like state writes as

$$
\begin{gathered}
g_{F}(x)=2 \sqrt{2 \pi} \xi^{3} \exp \left(\xi^{2} \varepsilon^{*}\right) * \\
*\left[\sum_{i=0}^{2} c_{i} \boldsymbol{\kappa}_{i}^{* 3 / 2} \exp \left(-\boldsymbol{\xi} \boldsymbol{\kappa}_{i}^{*} x\right)\right]^{2}
\end{gathered}
$$


Table 1 - Hartree-Fock coefficients and ionization energies forthe He-like sequence. (Clementi-Roetti[23]) $\Phi(\mathrm{r})=2 \sum_{\mathrm{i}=0}^{2} \mathrm{c}_{\mathrm{i}} \mathrm{k}_{\mathrm{i}}^{3 / 2} \mathrm{e}^{-\mathrm{k}_{\mathrm{i}} \mathrm{r}}$

\begin{tabular}{|c|c|c|c|c|c|c|c|}
\hline $\mathbf{Z}$ & $\mathbf{k}_{\mathbf{0}}$ & $\mathbf{k}_{\mathbf{1}}$ & $\mathbf{k}_{\mathbf{2}}$ & $\mathbf{c}_{\mathbf{0}}$ & $\mathbf{c}_{\mathbf{1}}$ & $\mathbf{c}_{\mathbf{2}}$ & $\mathbf{z}(\mathbf{a . u})$. \\
\hline & & & & & & & \\
\hline 2 & 1.45286 & 2.77954 & 4.34600 & .82958 & .18334 & .00824 & .91795 \\
\hline 3 & 2.45055 & 4.57259 & 6.67032 & .89066 & .12328 & .00088 & 2.79226 \\
\hline 4 & 3.43071 & 5.63150 & 7.35143 & .89855 & .09068 & .02158 & 5.66712 \\
\hline 5 & 4.44422 & 74.90274 & 11.31380 & .93036 & .07786 & .00013 & 9.54194 \\
\hline 6 & 5.44726 & 9.80425 & 14.61460 & .94428 & .06382 & -.00125 & 14.41690 \\
\hline 7 & 6.45215 & 11.69880 & 19.74410 & .95445 & .05228 & -.00096 & 20.29181 \\
\hline 8 & 7.45601 & 13.66210 & 22.59050 & .96175 & .01115 & -.00121 & 27.16675 \\
\hline & & & & & & & \\
\hline 26 & 25.45370 & 47.42210 & 56.67690 & .98817 & .01692 & -.00361 & 321.91659 \\
\hline
\end{tabular}

Next, we have the $n, \ell$ ) excited states, which we intentionally limit to $\ell=0,1$ and 2 and explain with radial $\mathrm{H}$-like wave functions ( $\omega \mathrm{f})$

$$
\begin{gathered}
R_{n \ell}(r)= \\
=\frac{2 Z_{1}^{3 / 2}}{n^{* 2}}\left[\frac{(n-\ell-1) !\left(2+2 a_{b}\right)_{n-\ell-1}}{\Gamma\left(2+2 a_{b}\right)}\right]^{1 / 2}\left(\frac{2 Z_{1} r}{n^{*}}\right)^{a_{b}} \times \\
\times \sum_{j=0}^{n-\ell-1} \frac{\left(-2 Z_{1} r / n^{*}\right)^{j}}{(n-\ell-1-j) !\left(2+2 a_{b}\right)_{j} j !}
\end{gathered}
$$

indexed with the explained below parameter (see Appendix) [20] $\mathrm{a}_{\mathrm{b}}$ dependent on $\mathrm{Z}, \mathrm{n}$ and $\ell$. So, the effective quantum number $n^{*}=n-\ell+a_{b}$, allows to explain excited bound state energies within the $\mathrm{H}$ like expression

$$
\mathrm{E}_{\mathrm{n} \ell}=-\frac{\mathrm{Z}_{1}^{2}}{\mathrm{n}^{* 2}}
$$

Given partial correlation contributing to $\mathrm{g}_{\mathrm{L}}(\mathrm{r})$ may now be given an explicit dimensionless expression,

$$
\begin{gathered}
g_{L \ell \leq 2}(x)=2 \sqrt{2 \pi} \xi^{3} \sum_{n=n \ell}^{\infty}(2 \ell+1)(2 \xi x)^{2 a_{b}} \times \\
\times \frac{\Gamma\left(n+2 a_{b}-\ell+1\right)(n-\ell-1) !}{n^{* 4+2 a} b} \times \\
\times \exp \left(\frac{\xi^{2}}{2 n^{* 2}}-\frac{2 \xi x}{n^{*}}\right) * \\
*\left[\sum_{j=0}^{n-\ell-1} \frac{\left(-2 \xi x / n^{*}\right)^{j}}{(n-\ell-1-j) ! \Gamma\left(2+2 a_{b}+j\right) j !}\right]^{2}
\end{gathered}
$$

where $\mathrm{n}_{\ell}=2$ for $\ell=0,1$ and $\mathrm{n}_{\ell}=3$ for $\ell=2$.

Terms pertaining to excited states with $\ell \geq 3$ are taken hydrogenic [25] and explained with $a_{b}=0$ through $\mathrm{H}-$ like $\mathrm{R}_{\mathrm{n} \ell}(\mathrm{r})$ for $\mathrm{Z}_{1}$.

\subsection{Diffusion state contributions}

As far as two-body e- $Z_{1}$ continuous states are considered we consistently restrict the $\ell \geq 3$ to pure Coulomb ones with charge $Z_{1}\left(a_{c}=0\right.$ in the sequel). For relative angular momenta $\ell \leq 2$, and positive relative energy e- $Z_{1}$. For relative angular momenta $\ell \leq 2$, and positive we now have to consider [24]

$$
R_{k \ell}(r)=\sqrt{\frac{2 k}{r}} \frac{\Pi_{s=1}^{\ell}\left(1+s^{2} / \eta^{2}\right)^{1 / 2}}{(1-\exp (-2 \pi \eta))^{1 / 2}} \eta^{a_{c}-\ell}\left(\frac { \Gamma ( 1 + a _ { c } - i \eta ) } { \Gamma ( 1 + \ell ) - i \eta } \left(x \sum_{p=0}^{\infty}(-1){ }^{p}\left(\frac{k^{2} r}{2 Z_{1}}\right)^{p}\left\{A_{2 p} J_{1+2 a_{c}+2 p}(\rho)-\frac{\rho}{2} B_{2 p+1} J_{2+2 a_{c}+2 p}(\rho)\right\}\right.\right.
$$

where $\eta=Z_{1} / k$, while $a_{c}$ relying on $Z, \rho$ and $k$ features the other positive energy and crucial QDM parameter (See Appendix).

Coefficients $A_{2 p}$ and $B_{2 p+1}$ are detailed as

$$
\begin{aligned}
& \mathrm{A}_{0}=1, \quad \mathrm{~A}_{2}=1+\mathrm{a}_{\mathrm{c}}, \quad \mathrm{A}_{4}=\left(1+\mathrm{a}_{\mathrm{c}}\right)\left(2+\mathrm{a}_{\mathrm{c}}\right) / 2 \\
& \mathrm{~B}_{1}=0, \mathrm{~B}_{3}=1 / 3, \quad \mathrm{~B}_{5}=\left(8+5 \mathrm{a}_{\mathrm{c}}\right) / 15,
\end{aligned}
$$

$$
\mathrm{p} \geq 3:
$$




$$
\begin{gathered}
\mathrm{p}(2 \mathrm{p}-3) \mathrm{A}_{2 \mathrm{p}}=\left[(\mathrm{p}-1)\left(2 \mathrm{p}-3+2 \mathrm{a}_{\mathrm{c}}\right)+(2 \mathrm{p}-3)\right. \\
\left.\left(\mathrm{p}+\mathrm{a}_{\mathrm{c}}\right)\right] \mathrm{A}_{2 \mathrm{p}-2}-\left(2 \mathrm{p}-3+2 \mathrm{a}_{\mathrm{c}}\right)\left(\mathrm{p}-1+\mathrm{a}_{\mathrm{c}}\right) \mathrm{A}_{2 \mathrm{p}-4-2 \mathrm{~h}^{2} \mathrm{~A}_{2 \mathrm{p}-6}} \\
(\mathrm{p}-1)(2 \mathrm{p}+1) \mathrm{B}_{2 \mathrm{p}+1}=\left[(\mathrm{p}-1)\left(2 \mathrm{p}+1+2 \mathrm{a}_{\mathrm{c}}\right)+\right. \\
\left.+(2 \mathrm{p}-1)\left(\mathrm{p}-1+\mathrm{a}_{\mathrm{c}}\right)\right] \mathrm{B}_{2 \mathrm{p}-1}-\left(2 \mathrm{p}-1+2 \mathrm{a}_{\mathrm{c}}\right) \\
\left(\mathrm{p}-1+\mathrm{a}_{\mathrm{c}}\right) \mathrm{B}_{2 \mathrm{p}-3-2 \mathrm{~h}^{2} \mathrm{~B}_{2 \mathrm{p}-5}}
\end{gathered}
$$

The resulting partial-correlation function

$$
\begin{gathered}
g_{D \ell}(r)=\frac{1}{4 \pi}\left(\frac{2 \pi \beta \hbar^{2}}{m}\right)^{3 / 2}(2 \ell+1) \times \\
\times\left(\int_{0}^{\infty} \exp \left(\frac{-\beta \hbar^{2} k^{2}}{2 m}\right)\left\{R_{k \ell}(r)\right\}^{2} d k\right.
\end{gathered}
$$

then appears through $\mathrm{y}=\xi \mathrm{k} / \mathrm{Z}_{1}$ under the form

$$
\begin{aligned}
& g_{D \ell \leq 2}(x)=\frac{\sqrt{2 \pi}}{x}(2 \ell+1)\left(\int_{0}^{\infty} d y \exp \left(\frac{-y^{2}}{2}\right) C(y, \xi) \times\right. \\
& \times\left[\sum_{p=0}^{\infty}(-1)^{p}\left(\frac{y^{2} x}{2 \xi}\right)^{p}\left\{A_{2 p} J_{v}(\rho)-\frac{\rho}{2} B_{2 p+1} J_{v+1}(\rho)\right\}\right]^{2}
\end{aligned}
$$

where $v=1+2 a_{c}+2 p$ with $\rho=2 \sqrt{2 \xi x}$ and

$$
\begin{aligned}
C(y, \boldsymbol{\xi})= & y \frac{\prod_{s=1}^{\ell}\left(1+s^{2} y^{2} / \xi^{2}\right)}{1-\exp (-2 \pi \xi / y)}\left(\frac{y}{\xi}\right)^{2\left(a_{c}-\ell\right)} \times \\
& \times\left(\frac{\Gamma\left(1+a_{c}-i \xi / y\right)}{\Gamma(1+\ell-i \xi / y)}\right)^{2}
\end{aligned}
$$

At this level of presentation, it should be appreciated that the displayed QDM formalism entirely relies on the two parameters $a_{b}$ and $a_{c}$. So, it is now timely to recall that $a_{b}$ is derived from the very accurate $(n, \ell)$ ionization energies $[20]$ while $a_{c}$ is directly related to the departure from the Coulomb phase, which we denote $\delta_{1}\left(\mathrm{k}^{2}\right)$ by [18]

$$
\begin{gathered}
\delta_{1}\left(k^{2)}=\arg \Gamma\left(1+a_{c}-i Z_{1} / k\right)-\right. \\
-\arg \Gamma\left(1+\ell-i Z_{1} / k\right)+\frac{1}{2} \pi\left(\ell-a_{c}\right)
\end{gathered}
$$

also a very well documented quantity. Our former assertion about the $a_{b}=a_{c}=0$ approximation for the $\ell \geq 3$ excited levels stems from detailed investigations of the $Z=2$ polarization model [25].

\subsection{Spin effects}

In hydrogenic systems, electron spin plays only a minim role essentially limited to relativistic calculations of the fine structure constant, pertaining relativistic modifications remaining small relative to the Bohr and non relativistic binding energy. The situation looks different in the He-sequence, because the total spin $S=0$ or 1 has to be included in the evaluations of $E_{n \ell}$ and $\delta_{\ell}\left(k^{2}\right)$. Energies of singlet and triplet states thus differ at most by 20 percent for the first excited states. As for as the present QDM framework is considered, this S-dependence is included within $a_{b}(n, \ell, Z, S)$ and $a_{c}(n, \ell, Z, S)$ with $\ell \leq 2$. (Practically, it suffices to include that $S$ dependence for low lying $\ell \leq 2$ levels). To determine those S-dependent states, were are thus led to envision a model with an electron orbiting in a field of a non-point-like ion, so the given wave functions do not exhibit the usual an antisymmetric form, and are distinct according to $\mathrm{S}=0$ or 1 . Spin effects are then accounted through

$$
\mathrm{g}_{\ell \leq 2}^{\overline{\mathrm{S}}}(\mathrm{x})=\frac{3}{4} \mathrm{~g}_{\ell \leq 2}^{\mathrm{S}=1}(\mathrm{x})+\frac{1}{4} \mathrm{~g}_{\ell \leq 2}^{\mathrm{S}=0}(\mathrm{x})
$$

leading to the more accurate expression

$$
\mathrm{g}(\mathrm{x})=\mathrm{g}_{\mathrm{F}}(\mathrm{x})+\sum_{\ell=0}^{2} \mathrm{~g}_{\ell} \overline{\mathrm{s}}(\mathrm{x})+\sum_{\ell>3} \mathrm{~g}_{\ell}(\mathrm{x})
$$

for the total $\mathrm{e}-\mathrm{Z}_{1}$ correlation function.

These spin effects decay with increasing $Z_{1}$. They are mostly significant for $Z=2$, with significant $\mathrm{S}=0,1$ discrepancies. Fig. 1 a display $\ell=0,1$ correlation functions with $\mathrm{S}=0,1$ for $\mathrm{Z}=2$. The $\mathrm{S}$ discrepancy shows up as significant. Fig. $1 b$ also displays same $g_{\square}(x)$ contrasted to those of $Z=4$ (boron). Considered temperatures feature same parameter for both elements. So, one witnesses that this S-effect decreases when $\mathrm{Z}$ increases, whenever the same ionization is maintained.

\subsection{Structure Effects}

Our presently used QDM techniques imply that the pair $e-Z_{1}$ correlation function be computed through hydrogen-like wave functions with net charge $Z_{1}=Z-1$. The structure effects featured by the He-sequence are expressed with non integer $a_{b}$ and $\mathrm{a}_{\mathrm{c}}$ parameters. The latter highlight the discrepancy 
between He-like $\mathrm{g}(\mathrm{x})$ and H-like ones with equivalent point like charge. Figs. 2 exhibit partial $\mathrm{g}_{\ell}(\mathrm{x})$ with $\ell=0,1$ or 2 for $\mathrm{He}$, then contrasted to their $\mathrm{H}$-like homologues.
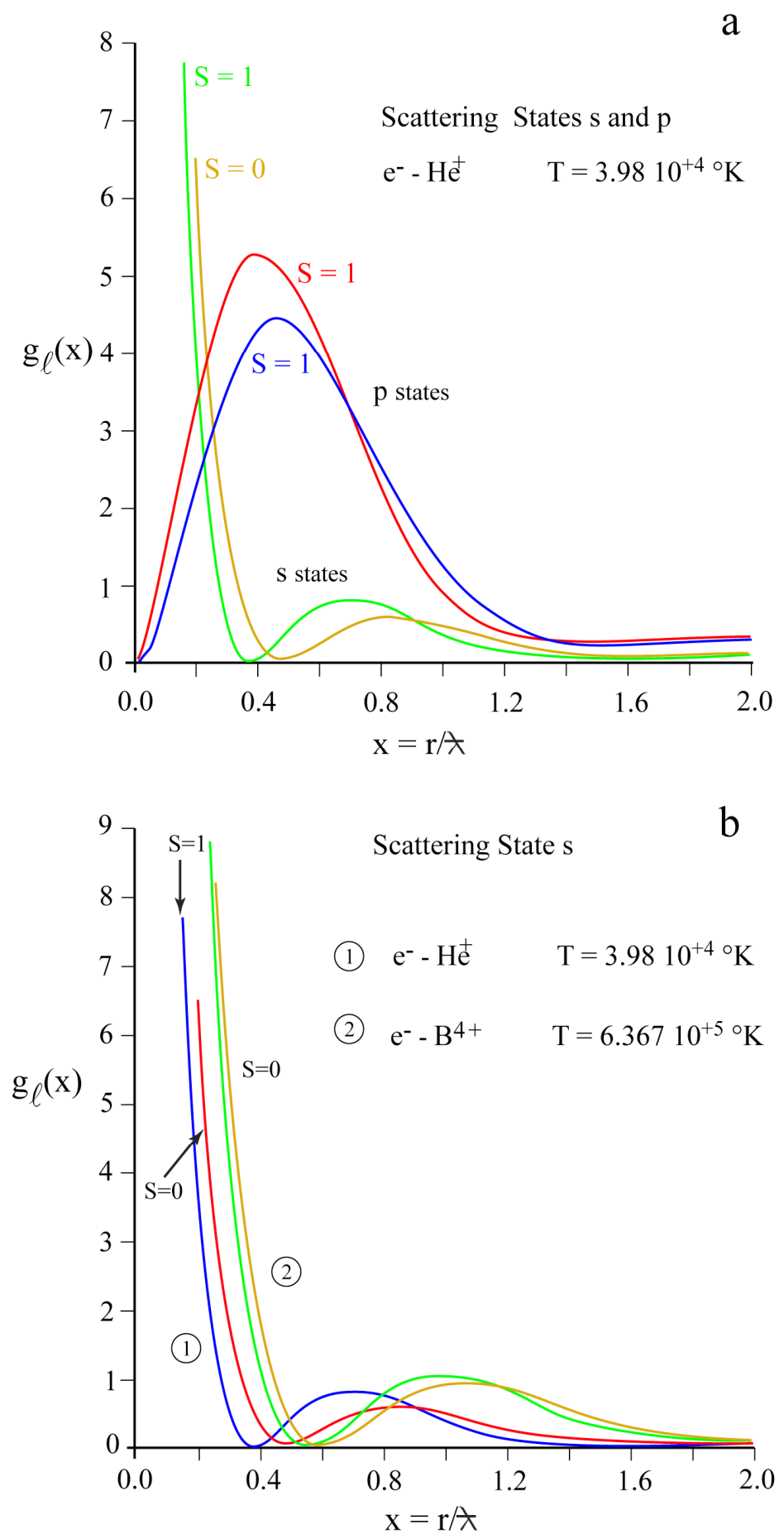

Figure 1 - Partial correlation function $\mathrm{g}_{\ell}(\mathrm{x})$ in terms of scattering states $\ell=0.1$ for total spin $\mathrm{s}=0.1$ : a) He 3-body plasma; b) He 3 body plasma contrasted to He-like B plasma 

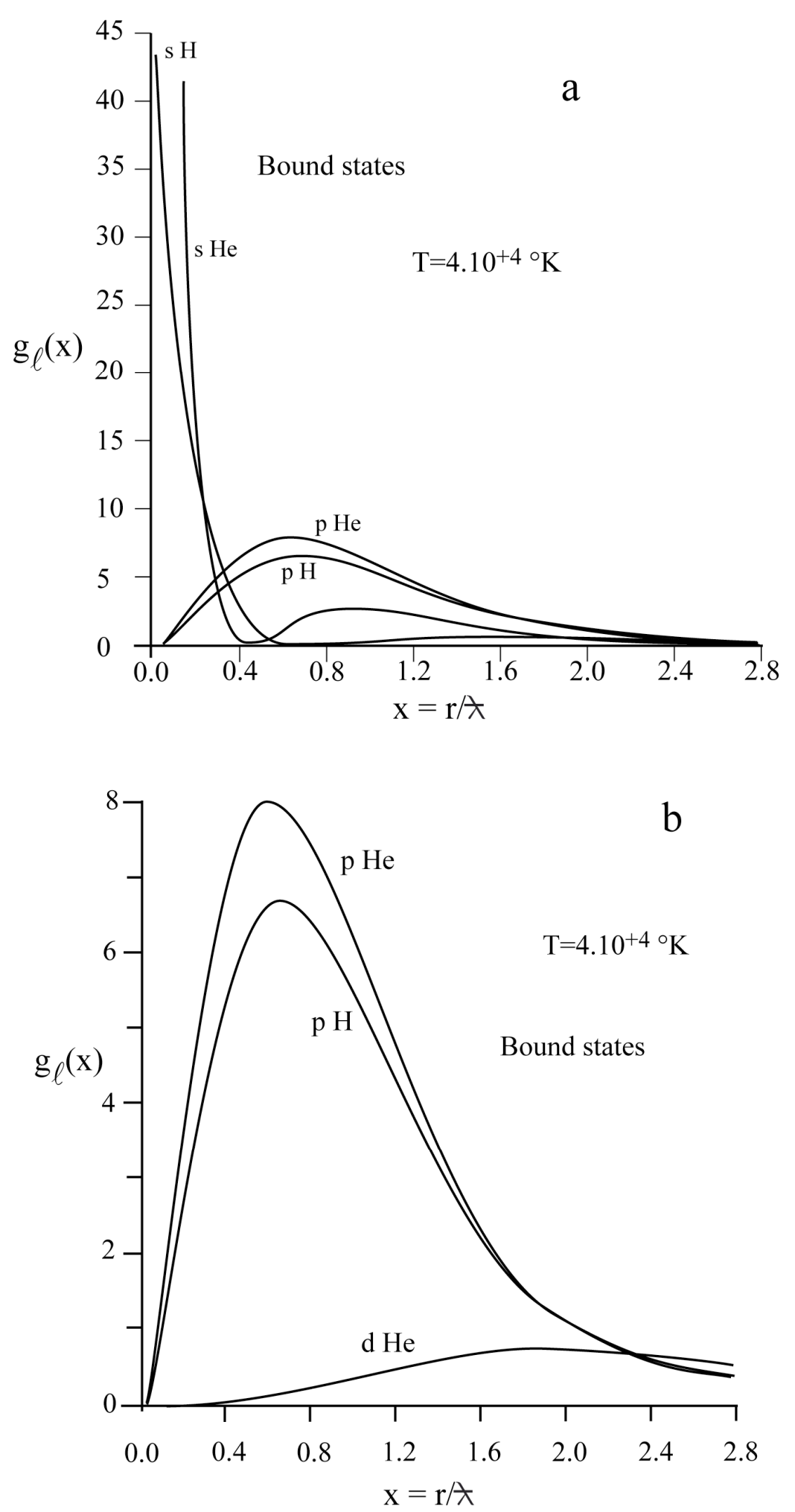

Figure 2 - Partial correlation functions out of bound states for $\mathrm{H}$ and $\mathrm{He}$ plasmas at $\mathrm{T}=4 \times 10^{4}{ }^{\circ} \mathrm{K}$ : a) $\ell=0.1$ for $\mathrm{H}$ and $\mathrm{He}$; b) $\ell=1$ for $\mathrm{H}$ and $\mathrm{He}$ and $\ell=2$ for $\mathrm{He}$ 


\section{Effective interaction $\mathrm{e}-\mathrm{Z1}$}

\subsection{General trends}

Elaborating upon the above detailed evaluation of the total e- $Z_{1}$ correlation function of

$$
\begin{gathered}
g(r)=g_{F}(r)+g_{D \ell \leq 2}(r)+ \\
+g_{L \ell \geq 3}(r)+g_{D \ell \leq 2}(r)+g_{D \ell \geq 3}(r)
\end{gathered}
$$

were are now entitled to proceed to the extraction of the $\mathrm{T}$-dependent $\mathrm{e}-\mathrm{Z}_{1}$ effective interaction from $\mathrm{Eq}$. (1). A first and instructive study concerns the comparison of $\mathrm{H}$-like $\mathrm{g}^{(\mathrm{H})}(\mathrm{x})$ and He-like $\mathrm{g}^{(\mathrm{He})}(\mathrm{x})$, with $\mathrm{x}=\mathrm{r} / \boldsymbol{\lambda}$

To implement this matching it is appropriate to take the e- $Z_{1}$ effective interaction under the form

$$
-\frac{\xi}{(Z-1)^{2}} u(x)=\frac{1}{\xi} \ln [g(x)]
$$

depicted on Fig. 3 for $\xi=2$ and the He-like sequence $2 \leq \mathrm{Z} \leq 26$. He-like $\mathrm{u}(\mathrm{x})$ gets closer to its H-like point-like limit when $\mathrm{Z}$ increases. Moreover, $\mathrm{g}^{(\mathrm{He})}(\mathrm{x})$ values are directly contrasted to $\mathrm{g}^{(\mathrm{H})}(\mathrm{x})$ values on Figure 4,

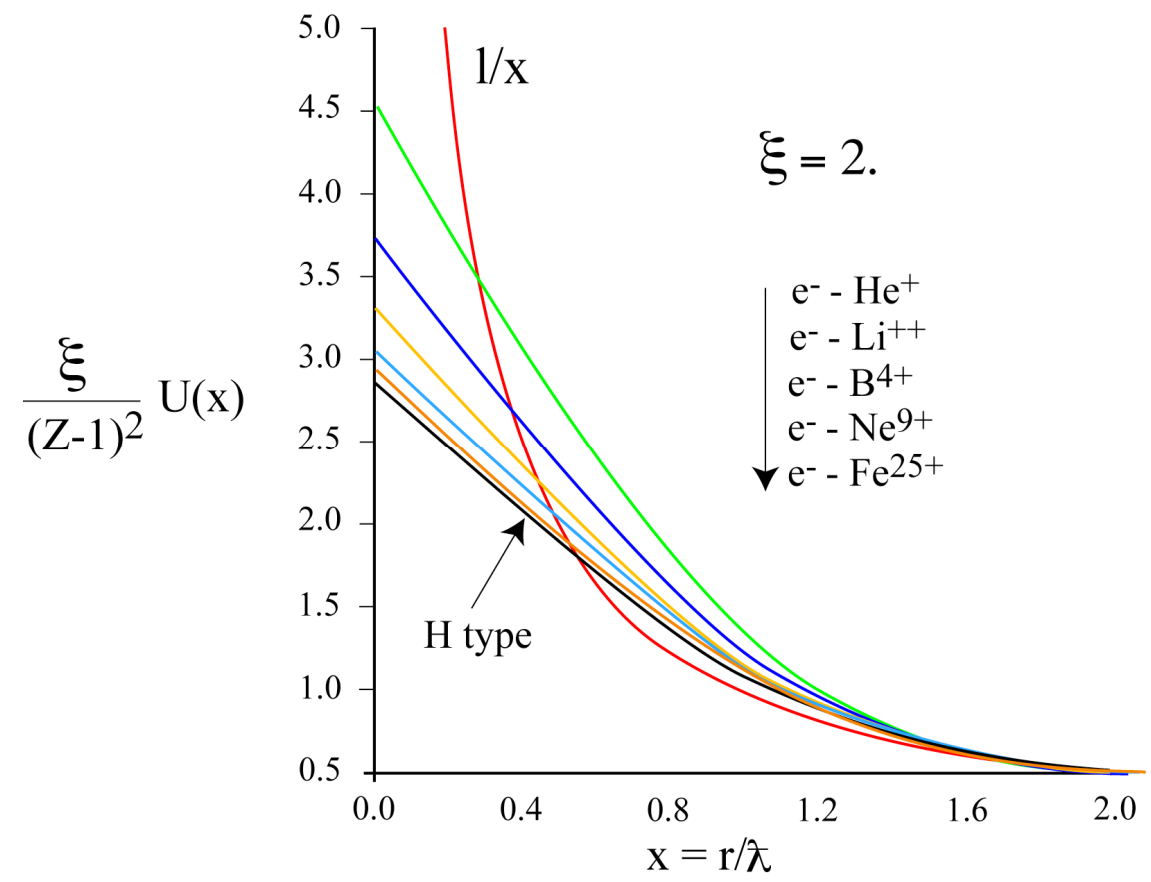

Figure 3- $-\frac{\xi}{(Z-1)^{2}} u(x)=\frac{1}{\xi} \ln [g(x)]$ for $\xi=2$ and He-like plasmas with $2 \leq \mathrm{Z} \leq 26$

through

$$
-\frac{\xi}{(Z-1)^{2}} u^{H e}(x)+\frac{\xi}{(Z-1)^{2}} u^{H}(x)=\frac{1}{\xi} \ln \left[g^{H e}(x) / g^{H}(x)\right]
$$

when $\xi$ is fixed, a given $T$ value is ascribed to a given $\mathrm{Z}$ value. One thus compares correlation functions at different $\mathrm{Z}$ values and equivalent ionization stage. 


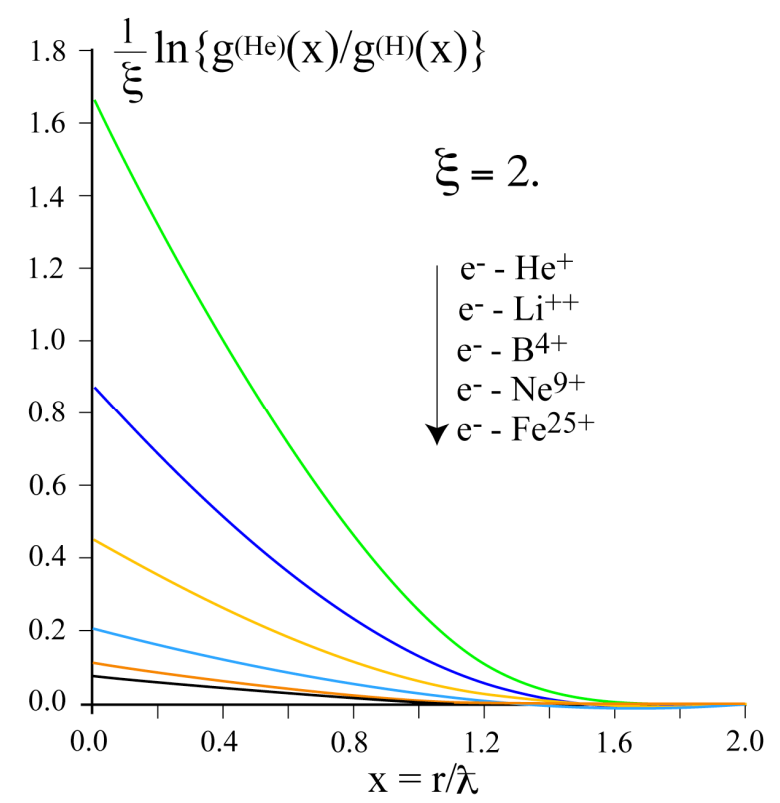

Figure 4 - He-H discrepancies $-\frac{\xi}{(Z-1)^{2}} u^{H e}(x)+\frac{\xi}{(Z-1)^{2}} u^{H}(x)=\frac{1}{\xi} \ln \left[g^{H e}(x) / g^{H}(x)\right]$

\subsection{Analytic expressions}

In order to secure a transparent and instructive comparison with standard effective interactions currently used in hydrogenic plasmas, we try to put the present He-like pseudopotential under a form as close as possible to the finite $\mathrm{T}$ extension of the Kelbg-Deutsch potential [2,4-6] as proposed by Gombert and Wagenknecht et al. [7]. Toward this goal, we thus recall the Kelbg expression $\left(\xi_{\mathrm{H}}=\mathrm{Z} \beta^{1 / 2}\right)[2]$

$$
\begin{gathered}
-\frac{\xi_{H}}{Z^{2}} U_{K}(x)=\frac{1}{x}\left[1-\exp \left(-2 x^{2}\right)\right]+ \\
+\sqrt{2 \pi}[1-\operatorname{Erf}(\sqrt{2} x)]
\end{gathered}
$$

in terms of the Error function

$$
\begin{gathered}
\operatorname{Erf}(x)=\frac{2}{\sqrt{\pi}}\left(_{0}^{x} \exp \left(-t^{2}\right) d t=\right. \\
=\frac{2}{\sqrt{\pi}} \sum_{n=0}^{\infty} \frac{(-1)^{n} x^{2 n+1}}{n !(2 n+1)}
\end{gathered}
$$

yielding $-\frac{\xi_{H}}{Z^{2}} U k(0)=\sqrt{2 \pi}$ and a $Z$-independent slope at the origin without $\mathrm{x}^{2}$ term in the small $\mathrm{x}$ expansion. A very closed related expression to $\mathrm{U}_{\mathrm{K}}(\mathrm{x})$ features the simpler Deutsch expression [4-6]

$$
-\frac{\xi_{\mathrm{H}}}{\mathrm{Z}^{2}} \mathrm{U}_{\mathrm{D}}(\mathrm{x})=\frac{1}{\mathrm{x}}\left(1-\mathrm{e}^{-\sqrt{2 \pi} \mathrm{x}}\right)
$$

Both $U_{K}(x)$ and $U_{D}(x)$ are mostly accurate in hydrogenic plasmas at very high (if not infinite) temperature. To remedy for this drawback Gombert and Wagenknecht et al [7] came up with the finite T-extension

$$
\begin{gathered}
-\frac{\xi_{H}}{Z^{2}} U_{G W}(x)=\frac{1}{x}\left[1-\exp \left(-2 x^{2}\right)\right]+ \\
+A_{H}\left[1-\operatorname{Erf}\left(\frac{2 \sqrt{\pi}}{A_{H}} x\right)\right]
\end{gathered}
$$

where

$$
\begin{gathered}
A_{H}=(2 \pi)^{\frac{1}{2}}+\pi\left(\frac{\pi}{3}-1\right) \xi+ \\
+2(2 \pi)^{\frac{1}{2}}\left(-\frac{\pi^{2}}{6}+\frac{\pi}{3}+\frac{\xi(3)}{2}\right) \xi^{2}+O\left(\xi^{3}\right)
\end{gathered}
$$

with the small $\mathrm{x}$-expansion

$$
-\frac{\xi}{\mathrm{Z}^{2}} \mathrm{U}_{\mathrm{GW}}(\mathrm{x})=\mathrm{A}_{\mathrm{H}}+2 \mathrm{x}-2\left(1-\frac{8 \pi}{3 \mathrm{~A}_{\mathrm{H}}^{2}}\right) \mathrm{x}^{3}+\ldots
$$


Now, we turn toward the He-like effective interactions fulfilling

$$
\begin{gathered}
\left(\xi_{\mathrm{He}}=(\mathrm{Z}-1) \beta^{1 / 2}=\xi_{\mathrm{H}} /(\alpha+1) \text { where } \alpha=1 /(\mathrm{Z}-1)\right) \\
=\frac{\xi_{H e}}{(Z-1)^{2}} U_{H e}(x)=-\frac{(\alpha+1)^{2} \xi_{H e}}{Z^{2}} U_{H e}(x)= \\
=\frac{1}{\xi_{H E}} \ln \left[g^{(H e)}(x)\right]
\end{gathered}
$$

and valid at any temperature $\mathrm{T}$, because bound and scattered states are explicitly included through the QDM approach. The given He-like effective interaction $\mathrm{U}_{\mathrm{He}}(\mathrm{x})$ is supposed to yield back $\mathrm{U}_{\mathrm{GW}}(\mathrm{x})$ for $\alpha=1 /(\mathrm{Z}-1) \rightarrow 0$.

First, $\mathrm{U}_{\mathrm{GW}}(\mathrm{x})$ gets validated for any finite temperature $\mathrm{T}$ with a $\mathrm{A}_{\mathrm{H}}$ parametrization for, $\xi \sim 1$ coincident with above expression (19) for $\xi<<1$. Then, we reach a hydrogenic pseudopotential valid at any $\mathrm{T}$. Next, we introduce an $\alpha$-parametrization acknowledging the He-like sequence (mostly $\mathrm{Z}=2 \rightarrow 26$ ). Then, we arrive at the $\alpha$-dependent expression

Table 2 - Coefficients involved in Eqs. (23a-b)

$$
\begin{gathered}
\frac{(\boldsymbol{\alpha}+1)^{2} \boldsymbol{\xi}}{Z^{2}} U_{H e}(x)=\frac{1}{x}\left[1-\exp \left(-2 x^{2}-\boldsymbol{\alpha} x^{3}\right)\right]+ \\
+A_{H e}\left[1-\operatorname{Erf}\left(B_{H e} x\right)\right] \\
A_{z} \equiv A_{H e} \equiv A(\boldsymbol{\xi}, \boldsymbol{\alpha})=A(\boldsymbol{\xi}, \boldsymbol{\alpha}=0) f(\boldsymbol{\alpha}) \\
A(\boldsymbol{\xi}, \boldsymbol{\alpha}=0)=\sqrt{2 \pi}+\boldsymbol{\xi} \frac{a_{0}+a_{1} \xi+a_{2} \xi^{2}}{1+a_{3} \boldsymbol{\xi}+a_{4} \boldsymbol{\xi}^{2}} \\
f(\boldsymbol{\alpha})=1+\boldsymbol{\alpha} \operatorname{Erf}[b \boldsymbol{\alpha}], \quad f(\boldsymbol{\alpha}=0)=1 \\
B_{H e} \equiv B(\xi, \alpha)=\frac{B(\boldsymbol{\xi}, \boldsymbol{\alpha}=0)}{g(\boldsymbol{\xi}, \boldsymbol{\alpha})} \\
B(\boldsymbol{\xi}, \boldsymbol{\alpha}=0)=\frac{2 \sqrt{\pi}}{A(\xi, \boldsymbol{\alpha}=0)}
\end{gathered}
$$

and

$$
\begin{gathered}
g(\xi, \alpha)=1+\xi^{2}\left(b_{0}+b_{1} \xi^{2}\right) \operatorname{Erf}\left[b_{2} \sqrt{\alpha}\right] \\
g(\xi, \alpha=0)=g(\xi=0, \alpha)=1
\end{gathered}
$$

Coefficients $a_{0}, a_{4}, a, b, b_{0}, b_{1}$ et $b_{2}$ are given on Table 2.

\begin{tabular}{|c|c|c|}
\hline $\mathrm{a}_{0}=.14827548$ & $\mathrm{a}_{1}=.0155383$ & $\mathrm{a}_{2}=.00421951$ \\
\hline $\mathrm{a}_{3}=.00027919$ & $\mathrm{a}_{4}=.0116586$ & \\
\hline $\mathrm{a}=1.75972$ & $\mathrm{~b}_{2}=.291829$ & \\
\hline $\mathrm{b}_{0}=.852678$ & $\mathrm{~b}_{1}=.0425476$ & $\mathrm{~b}_{2}=.00920166$ \\
\hline
\end{tabular}

Figure 5 feature A and B- $\xi$ variations in terms of $\alpha$ parameter. Curves with $\alpha=0$ pertain to $\mathrm{U}_{\mathrm{GW}}(\mathrm{x})$ interaction valid for any $\mathrm{T}$ value. Kelbg's parameters get retriwed for $A_{k}=\sqrt{2 \pi}$ and $B_{k}=\sqrt{2}$ at $\alpha=\xi=0$. A is obviously more $\alpha$-sensitive than
B. Permuting $\alpha$ and $\xi$ roles, we get the $\xi$ parametrized A and B in terms of $\alpha$, on Figure 6. We do not pay much attention to $\xi>4, A$ and $B$ values, featuring lower temperatures when H-like and He-like ions become scarce. 

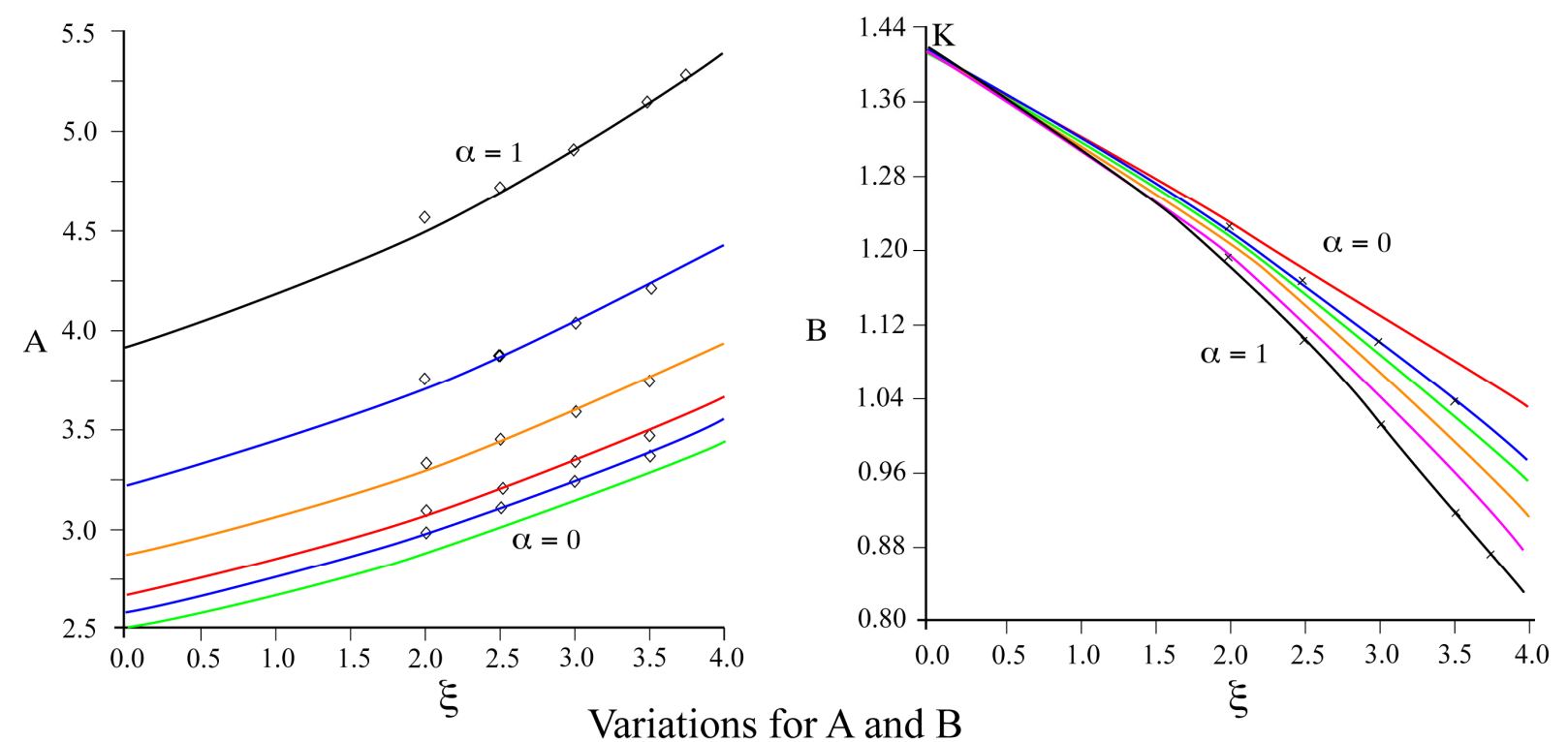

Figure 5 - A and B (Eqs.23 a,b) for He-like effective interactions in terms of $\xi$ and $0 \leq \alpha \leq 1$ respectively

$\mathrm{U}_{\mathrm{GW}}(\mathrm{x})$ data are also retrieved for $\xi<<1$ and $\alpha$ $=0$, with a very good accuracy. Numerically speaking, the present $\mathrm{U}_{\mathrm{He}}(\mathrm{x})$ fits the e- $\mathrm{Z}_{1}$ radial distribution function with a 1-2 percent precision. Near $\mathrm{x}=0$, it also fulfils

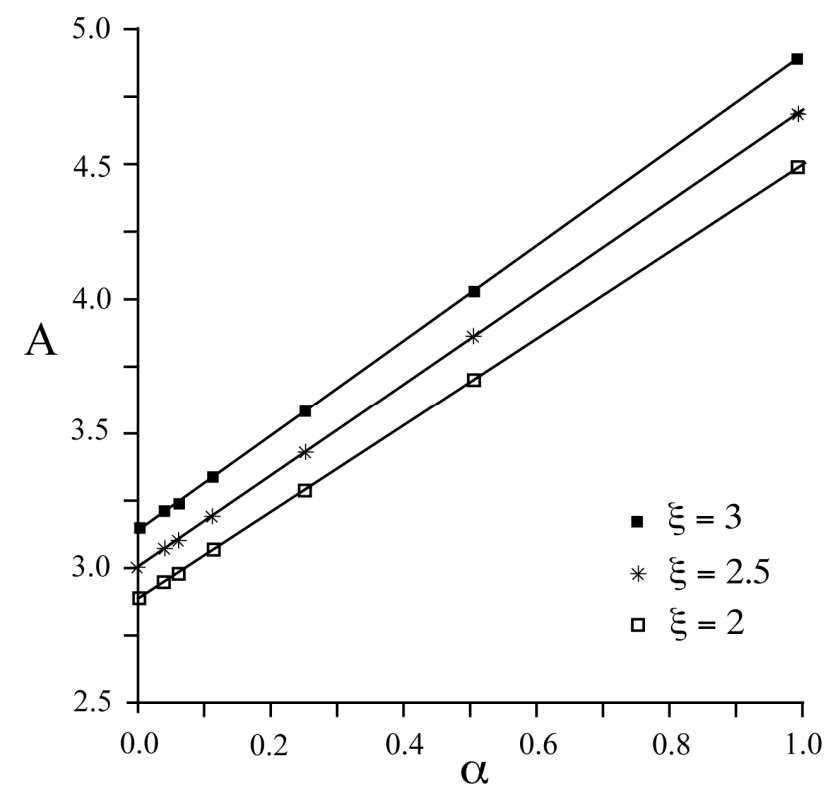

$$
\begin{gathered}
-\frac{(\alpha+1)^{2} \xi}{Z^{2}} U_{H e}(x) \cong A+2\left(1-\frac{A_{H e} B_{H e}}{\sqrt{\pi}}\right) x+ \\
+\boldsymbol{\alpha} x^{2}-2\left(1-\frac{A_{H e} B_{H e}^{3}}{3 \sqrt{\pi}}\right) x^{3}+\ldots \ldots
\end{gathered}
$$

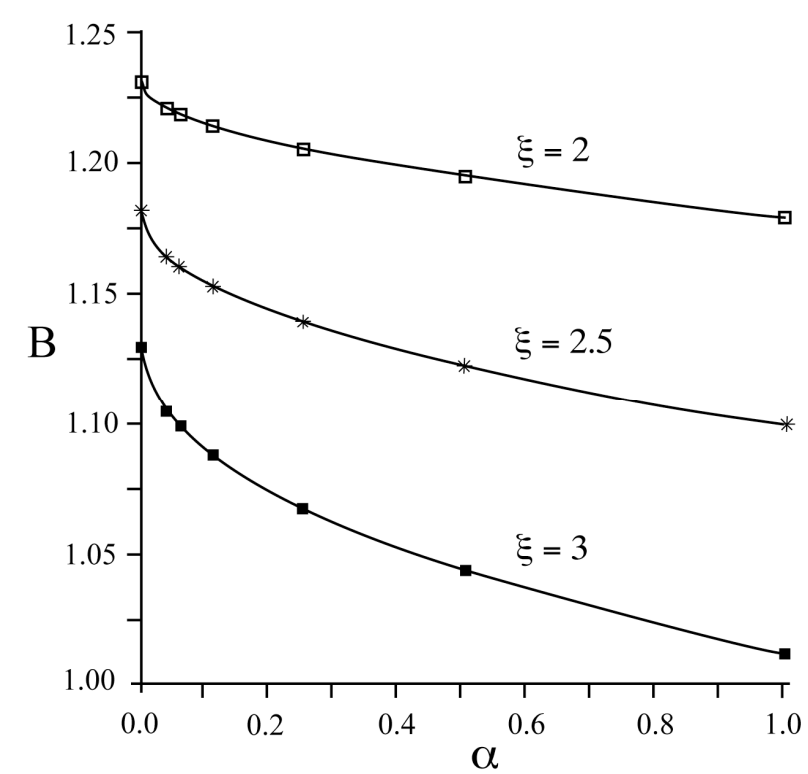

Figures 6 - A and B (Eq. (23a-b)) for $\mathrm{U}_{\mathrm{He}}(\mathrm{x})$ In terms of $0 \leq \alpha \leq 1$ and $2 \leq \xi \leq 3$ 
highlighting now an $\mathrm{x}^{2}$ term, absent in previous $\mathrm{H}$-like effective interactions. Moreover, the slope at $\mathrm{x}=0$

$$
\mathrm{P}_{\mathrm{He}}=2\left(1-2 \frac{\mathrm{f}(\alpha)}{\mathrm{g}(\xi, \alpha)}\right) \equiv \mathrm{P}_{0}(\xi, \alpha)
$$

where $\mathrm{P}(\xi, \alpha=0)=-2=\mathrm{P}_{\mathrm{H}}$ appears $\alpha$-and $\mathrm{T}$ dependent.

\subsection{Numerics [25]}

Figures $7 \mathrm{a}-\mathrm{c}$ feature the above displayed $\mathrm{e}-\mathrm{Z}_{1}$ effective interactions systematically confronted to their Hydrogen-like homologues for $Z=2,10$ and 92, respectively. The e- $Z_{1}$ distance is scaled by the electron thermal wavelength $\lambda$ in a dense plasma with $T_{e}=T_{i}$, where $T_{i}$ designates the same ion temperature for ion $\mathrm{Z}$ and ion $\mathrm{Z}-1$.

D denotes Deutsch expression (18), GW is for Gombert-Wagenknecht expression (19) and $\mathrm{K}$ is for Kelbg expression (16). The Helium-like effective interactions (23) are thus compared to their various Hydrogen-like counterparts $\mathrm{U}_{\mathrm{K}}(\mathrm{x})(\mathrm{Eq} 16), \mathrm{U}_{\mathrm{D}}(\mathrm{x})$ (Eq.18) and $\mathrm{U}_{\mathrm{GW}}(\mathrm{x})$ (Eq.19). The He-like $-\mathrm{H}$-like discrepancies are strongly noticeable for $\mathrm{x} \leq 1$, and increase with $Z$. These results demonstrate clearly that WDM properties computed for He-like Be, for instance, cannot be approximated by the H-like effective interaction $(16,18,19)$. In this connection, it is pretty instructive to test the low-T behavior of expression (23) with the dominant H-like approximation [27]

$$
g_{e Z_{1}}(x)=\log \left[2 \sqrt{\pi} \xi^{3} e^{\xi^{2} / 2-2 \xi x}\right] / \xi
$$

denoted as $\mathrm{H}_{\mathrm{bd}}$ in Figures 8 which lies pretty close to $\mathrm{U}_{\mathrm{GW}}(\mathrm{x})$ for $\mathrm{x} \leq 0.5$, while $\mathrm{g}_{\mathrm{eZ1}}(0) \sim \mathrm{U}_{\mathrm{GW}}(0)$ is well represented by $\log \left(g_{i j}(0, \gamma)\right)$ where $\gamma=2 \xi^{2}$ and

$$
g_{i j}(0, \gamma)=1+\sqrt{\pi \gamma}+\sum_{n=0}^{\infty} \frac{\Gamma\left(1+\frac{n}{2}\right)}{n !} \zeta(n+2) \gamma^{\frac{n}{2}+1}
$$

In terms of Gamma $\Gamma(\mathrm{x})$ and Zeta $\zeta(\mathrm{x})$ functions respectively. As $\mathrm{T}$ increase with no limits, the Helike behavior (23) tends to join the H-like homologues (16,18-19), with a marked saturation observed for $\mathrm{T}>\mathrm{J}$ (Ryd) with [28]

$$
\begin{aligned}
& J=Z_{1}^{2}-\frac{5}{4} Z_{1}+0.315311-0.01707 \frac{1}{Z_{1}}+ \\
& +0.00068 \frac{1}{Z_{1}^{2}}+0.00164 \frac{1}{Z_{1}^{3}}+0.00489 \frac{1}{Z_{1}^{4}},
\end{aligned}
$$

nonrelativistic ionization energy of ion $\mathrm{Z}_{1}$.

$\mathrm{Hbd}$ refers to the $\mathrm{H}$-like bound state contribution (26). D, GW and K attributions are the same as on Figures 7.

\section{Ionization in dense 3-component plasmas}

\subsection{Notations}

It is convenient to write the canonical ionization in a 3-component (e, ion $\mathrm{Z}$, ion $\mathrm{Z}_{1}$ ) plasma under the form

$$
v=\frac{\rho_{Z}}{\rho_{Z}+\rho_{Z_{1}}}=\frac{\rho_{Z}}{\rho I}
$$

Quasi-neutrality then features $\rho_{\mathrm{e}}=\mathrm{Z} \rho_{\mathrm{z}}+\mathrm{Z}_{1} \rho_{\mathrm{Zl}}$ with the Debye length $\lambda_{D}^{-2}=4 \pi \beta \rho_{I} Z\left(Z_{1}+2 v\right)$ and the diffraction parameter $\left(\bar{\lambda}=\frac{\hbar}{\sqrt{m_{e} k_{B} T}}\right)$ $\eta=\frac{\bar{\lambda}}{\lambda_{D}}$ fulfilling

$$
\eta^{2}=4 \pi \beta \rho_{I} \bar{\lambda}^{2} Z\left(Z_{1}+2 v\right)
$$

Moreover, the total density $\rho=(Z+x) \rho I$ allows to explain the plasma parameter as $(\xi=Z \bar{\lambda})$ under the form

$$
\Lambda=\frac{1}{4 \pi \rho \lambda_{D}^{3}}=\frac{Z_{1}+2 v}{Z+v} \eta \xi
$$

Numerically, one thus gets

$$
\begin{gathered}
\eta=4.32310^{-7}\left[Z\left(Z_{1}+2 v\right)\right]^{1 / 2} \frac{\rho_{I}^{1 / 2}\left(\mathrm{~cm}^{-3}\right)}{T^{3 / 2}\left({ }^{\circ} \mathrm{K}\right)} \\
\Lambda=2.4210^{-4} \frac{\left[Z\left(Z_{1}+2 v\right)\right]^{3 / 2}}{Z+\boldsymbol{v}} \frac{\boldsymbol{\rho}_{I}^{1 / 2}\left(\mathrm{~cm}^{-3}\right)}{T^{3 / 2}\left({ }^{\circ} \mathrm{K}\right)}
\end{gathered}
$$


(a)
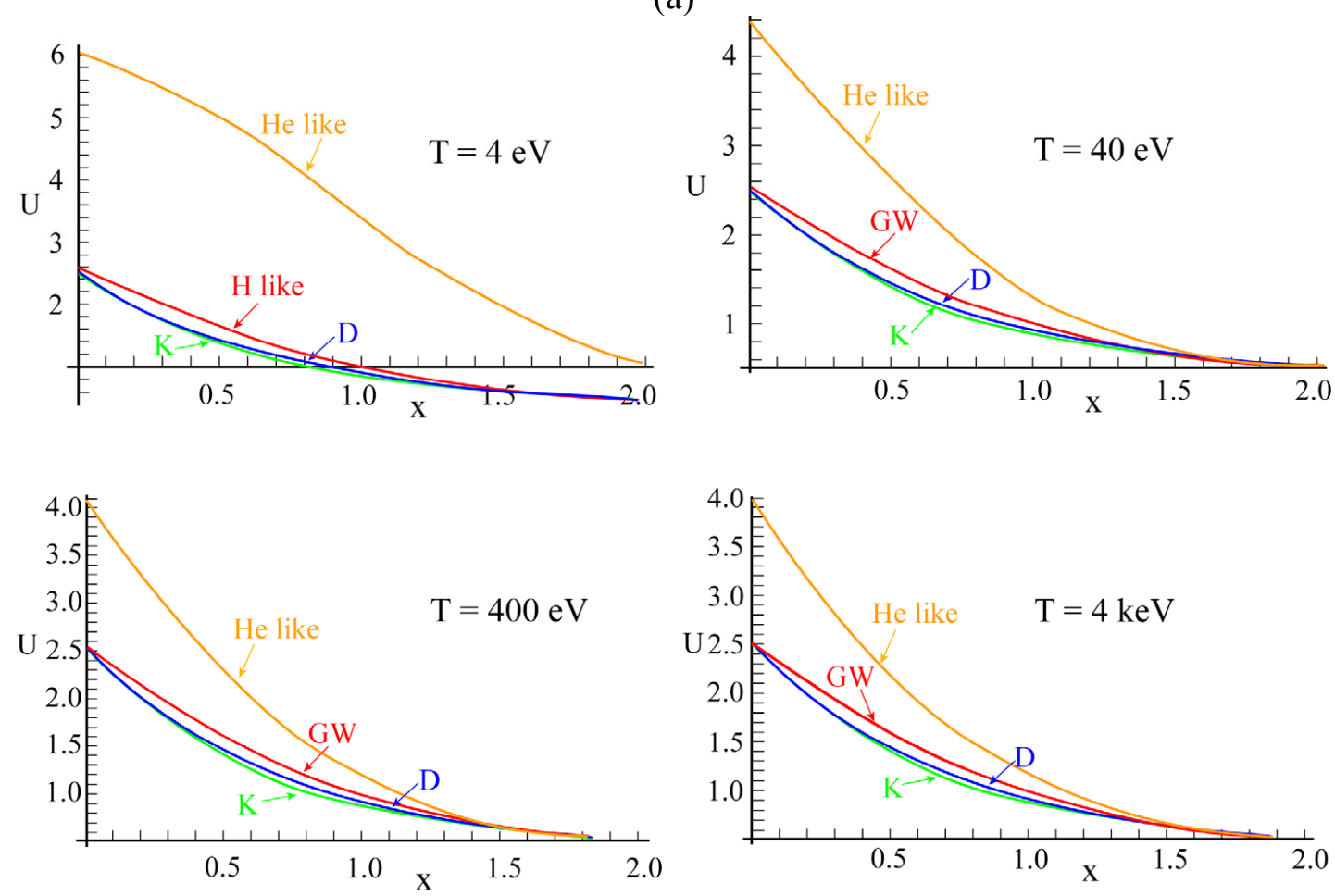

$$
Z=2
$$

(b)
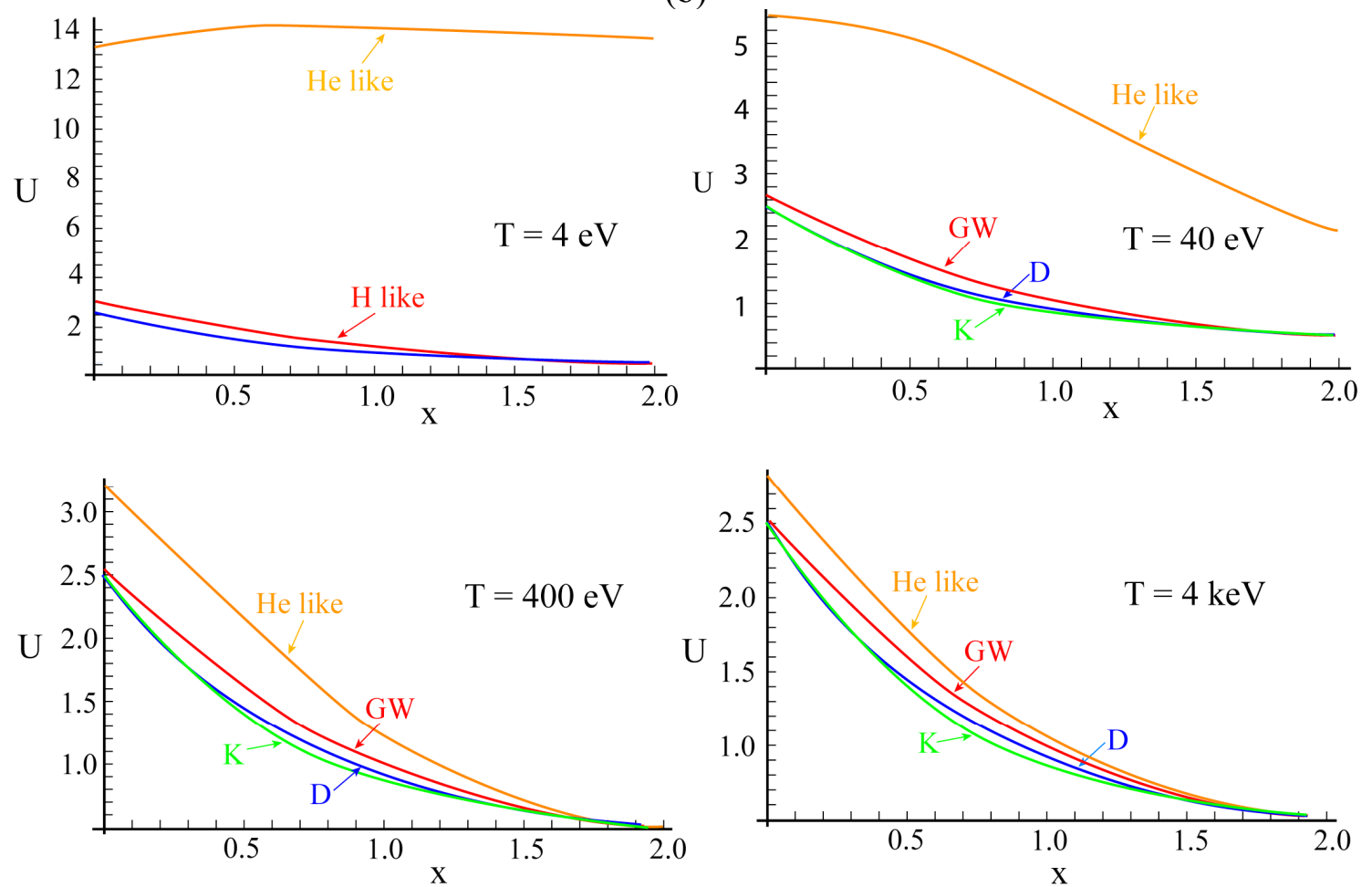

$$
Z=10
$$


(c)
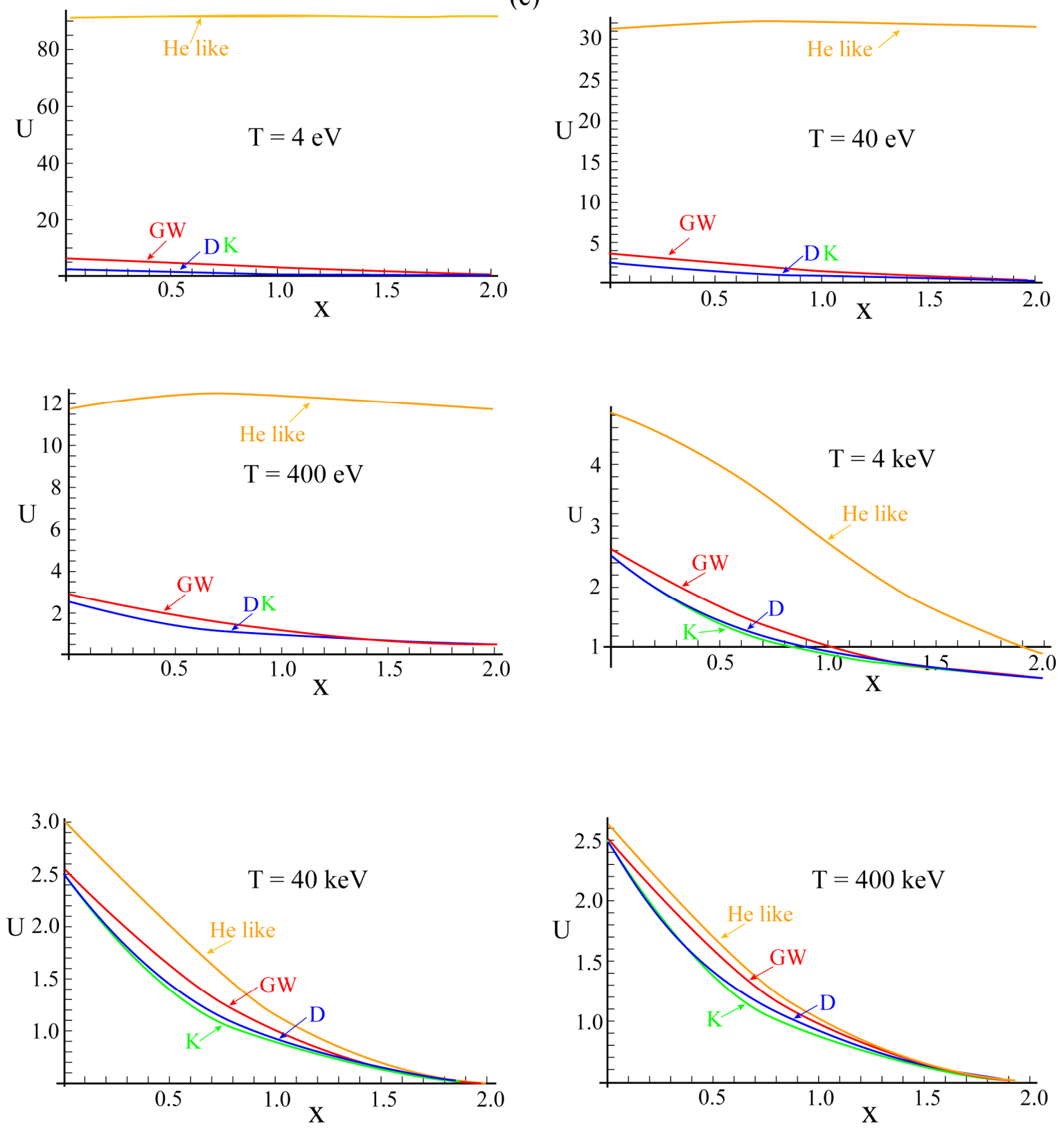

$$
Z=92
$$

Figures 7 - He-like effective interaction (23) contrasted to its $\mathrm{H}$-like homologues $(16,18,19)$ in a He plasma.

(a) $\mathrm{Z}=2$ for $4<\mathrm{T}(\mathrm{eV})<4000$

(b) (b). $Z=10$ for $4<\mathrm{T}(\mathrm{eV})<4000$

(c) $Z=92$ for $0.4<\mathrm{T}(\mathrm{keV})<400$ 

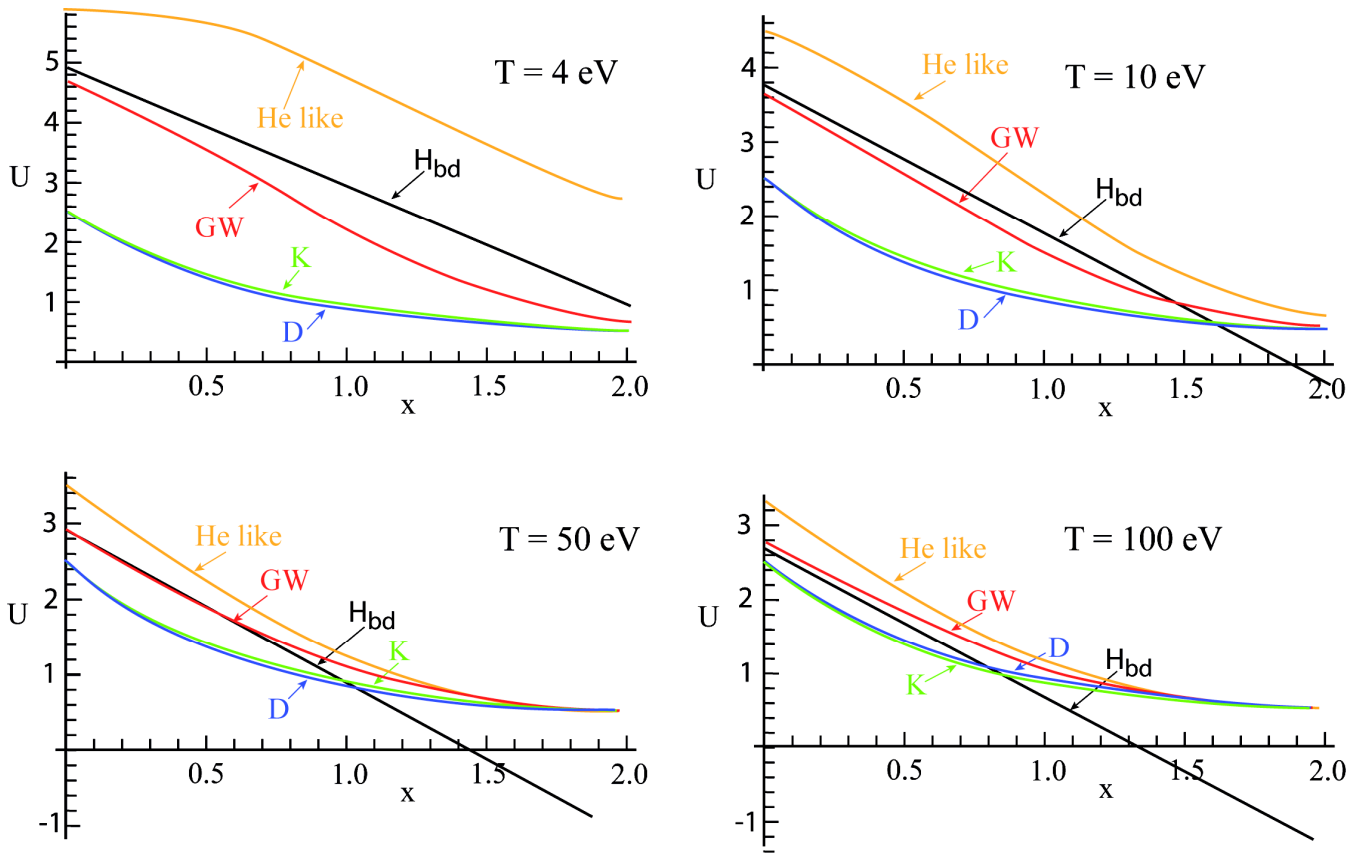

Figures 8 - He-like effective interaction (23) contrasted to its H-like homologues $(16,18,19)$ $U=-\frac{\varsigma_{H}}{Z^{2}} U_{G W, K, D}$ in a He plasma, with $\mathrm{Z}=4$

\subsection{Ionization equilibrium}

At equilibrium in a 3-component plasma the canonical free energy $F$ fulfills $\frac{d F}{d v}=0$ for a given $\left(\mathrm{T}, \rho_{\mathrm{I}}\right)$ pair. It then appears appropriate to split the given partial derivative as

$$
\frac{d F_{0}}{d \boldsymbol{\nu}}+\frac{d F_{e x}}{d \boldsymbol{v}}+\frac{d F_{c}}{d \boldsymbol{v}}+\frac{d F_{Q}}{d \boldsymbol{v}}=0
$$

First, the ideal contribution is explained through

$$
\frac{\beta}{V} \frac{d F_{0}}{d \boldsymbol{v}}=\rho_{I} \ln \left[\sqrt{\frac{\pi}{2}} \frac{\eta^{3}}{\Lambda} \frac{v\left(Z_{1}+v\right)}{(1-v)(Z+v)} \sigma(T)\right]
$$

with

$$
\begin{gathered}
\sigma(T)=\sum_{n=1}^{\infty} n^{2}\left[\exp \left(\boldsymbol{\beta} I_{Z_{1}} Z^{2} / n^{2}\right)-1-\frac{\beta I_{Z_{1}} Z^{2}}{n^{2}}\right] \\
I_{Z_{1}}=\frac{Z_{1}}{\boldsymbol{\eta}^{2}}
\end{gathered}
$$

then, we have the Coulomb part $\left(\beta=1 / \mathrm{k}_{\mathrm{B}} \mathrm{T}\right)$ and the exchange one

$$
\begin{gathered}
\frac{\beta}{V} \frac{d F_{c}}{d \boldsymbol{v}}=-\rho_{I} \Lambda \frac{Z+v}{Z_{1}+2 v} \\
\frac{\beta}{V} \frac{d F_{e x}}{d v}=\frac{\sqrt{\pi}}{8} \rho_{I} \frac{\eta^{3}}{\Lambda} \frac{Z_{1}+v}{Z+v}\left[1+\frac{\sqrt{\pi}}{12 \sqrt{3}} \frac{\eta^{3}}{\Lambda} \frac{Z_{1}+v}{Z+v}\right]
\end{gathered}
$$

Quantum corrections $\frac{\partial F_{Q}}{\partial v}$ are also split, so we get the first contribution under the form

$$
\frac{\boldsymbol{\beta}}{V} \frac{d F_{1}}{d \boldsymbol{v}}=\boldsymbol{\rho}_{I} \frac{\eta^{2}}{Z\left(Z_{1}+2 v\right)} x I_{1}
$$

with

$$
\begin{aligned}
I_{1}= & \left(Z_{1}+v\right)\left[-\frac{1}{2}+\frac{A_{e}^{4}}{12 \pi^{2}}\right]+Z\left(Z_{1}+2 v\left[\frac{1}{4}-\frac{A_{z}^{4}}{24 \pi^{2}}\right]\right)+ \\
& +Z_{1}\left(Z_{1}-1+2 v\right)\left[\frac{R_{0}(\boldsymbol{\alpha})}{4}-\frac{A_{Z_{1}}^{4}}{24 \pi^{2}} \int(x)\left[g\left(\xi_{1}, \alpha\right)\right]^{3}\right]
\end{aligned}
$$

In the above $A_{e}, A_{z}$ and $A_{z 1}$ feature respectively the three T-dependent effective interactions [30]

$$
u_{e z}(r)=-\frac{Z}{r}\left[1-e^{-2 r^{2} \bar{\lambda}^{2}}\right]-\frac{Z A(\xi)}{\bar{\lambda}}[1-\operatorname{erf}(B(\xi) r / \bar{\lambda})]
$$




$$
\begin{gathered}
u_{e e_{1}}(r)=-\frac{Z_{1}}{r}\left[1-e^{-2 r^{2} / \bar{\lambda}^{2}-\alpha r^{3} / \lambda^{-3}}\right]- \\
-\frac{Z_{1} A\left(\xi_{1}, \alpha\right)}{\bar{\lambda}}\left[1-\operatorname{erf}\left(B\left(\xi_{1}, \alpha\right) r / \bar{\lambda}\right)\right] \\
u_{e e}(r)=\frac{1}{r}\left[1-e^{-2 r^{2} / \lambda_{e}^{-2}}\right]+\frac{A\left(\xi_{e}\right)}{\bar{\lambda}_{e e}}\left[1-\operatorname{erf}\left(B\left(\xi_{e}\right) r / \bar{\lambda}_{e e}\right)\right]
\end{gathered}
$$

with $A_{e} \equiv A\left(\xi_{e}\right), A_{z} \equiv A(\xi)$ and $A_{Z_{1}}=A\left(\xi_{1}, \alpha\right)$ where $\xi=Z \bar{\lambda}, \xi_{1}=Z_{1} \bar{\lambda}$ and $\xi_{e}=\bar{\lambda}_{e e}=\sqrt{2} \bar{\lambda}$

Their, putting the Fourier transform of $\mathrm{U}_{\mathrm{ee}}(\mathrm{r})$ under the form $(y=k \lambda)$

$$
u_{e e}(y)=4 \pi \bar{\lambda}^{2}\left(\frac{1}{y^{2}}-\frac{1}{2} S_{e}\left(y, \boldsymbol{\xi}_{e}\right)\right),
$$

while explaining the determinant of the above three $\mathrm{U}_{\mathrm{ij}} \mathrm{S}^{\mathrm{S}}$ interactions in $\mathrm{k}$-space, as

$$
\begin{gathered}
D(k)=1+\beta \rho_{I}\left\{\left(Z_{1}+v\right) u_{e e}(k)+\left[Z^{2}(x)+Z_{1}^{2}(1-v)\right] \frac{4 \pi}{k^{2}}\right\}+ \\
+\beta^{2} \rho_{I}^{2}\left(Z_{1}+v\right)\left\{\left[Z^{2} x+Z_{1}^{2}(1-v)\right] u_{e e}(k) \frac{4 \pi}{k^{2}}-(1-v)\left[u_{e e_{1}(k)}\right]^{2}-v\left[u_{e z(k)}\right]^{2}\right\} \\
-\boldsymbol{\beta}^{3} \boldsymbol{\rho}_{I}^{3} \boldsymbol{v}(1-v)\left(Z_{1}+\boldsymbol{v}\right)\left[Z u_{e z_{1}}(k)-Z_{1} u_{e z}(k)\right]^{2} \frac{4 \pi}{k^{2}} \\
\sqrt{\frac{\pi}{2}} \frac{\eta^{3}}{\Lambda} \frac{v\left(Z_{1}+v\right)}{(1-v)(Z+v)} \sigma(T)=
\end{gathered}
$$

with Coulomb ion-ion interactions

$$
\begin{gathered}
u_{z z}(k)=Z^{2} u_{p p}(k), u_{z_{1} z_{1}}(k)=Z_{1}^{2} u_{p p}(k), \\
u_{z z_{1}}(k)=Z Z_{1} u_{p p}(k)
\end{gathered}
$$

and $\mathrm{u}_{\mathrm{pp}}(\mathrm{k})=\frac{4 \pi}{\mathrm{k}^{2}}$

$$
=\exp \left[f_{c}+f_{e x}+f_{Q 1}+f_{Q 2}\right]
$$

where $[29,30]$

$$
\begin{gathered}
f_{c}=\Lambda \frac{Z+v}{Z_{1}+2 v} \\
f_{e x}=\frac{\sqrt{\pi}}{8} \frac{\eta^{3}}{\Lambda} \frac{Z_{1}+v}{Z+v}\left[1+\frac{\sqrt{\pi}}{12 \sqrt{3}} \frac{\eta^{3}}{\Lambda} \frac{Z_{1}+v}{Z+v}\right]
\end{gathered}
$$

and

$$
\begin{gathered}
f_{Q 1}=-\frac{\eta^{3}}{Z\left(Z_{1}+2 v\right)} I_{1} \\
f_{Q 2}=-\frac{\Lambda}{\eta} \frac{1}{\pi} \frac{Z+v}{Z\left(Z_{1}+2 v\right)} I_{2}
\end{gathered}
$$

with $(y=k \lambda)$

$$
I_{2}=\int_{0}^{\infty} y^{2} d y\left[\frac{d D(y) / d x}{D(y)}-\frac{2 Z}{y^{2}+\eta^{2}}+\frac{1}{2} S_{e}\left(k, \boldsymbol{\xi}_{e}\right)\right]
$$

Finally, the above $\mathrm{dF} / \mathrm{d} v=0$ relation boils down to

\subsection{Numerical results}

Conspicuous dependences of $v$ on $\rho_{1}=\rho_{z}+\rho_{z 1}$ are detailed on Figs. $9 a-b$, for $\mathrm{He}(Z=2)$ and $C(Z=6)$ plasmas. The left and decaying portion which is mostly $\mathrm{T}$-dependent of $\mathrm{v}$ versus $\rho_{1}$ shows a rather weak $\alpha$-dependence, especially for Carbon, while 
the increasing density-dependent portion appears strongly $\alpha$-resolved. This second behavior documents the fact that in a very dense plasma medium the nonpointlike structure of ion $Z_{1}$ is quantitatively perceived.

Moreover, for both $\mathrm{Z}=2$ and $6, \alpha \neq 0$ produces the smallest ionization, while the bare Coulomb interaction yields the highest. The ionization patterns featured on Figs. 9 show clearly that even a finite- $T$ effective $e-Z$ and hydrogenic interaction would not be able to reproduce them. These outputs demand the implementation of an accurate albeit analytic effective quantum defect approach as evidence by Eqs. $(6,10)$.
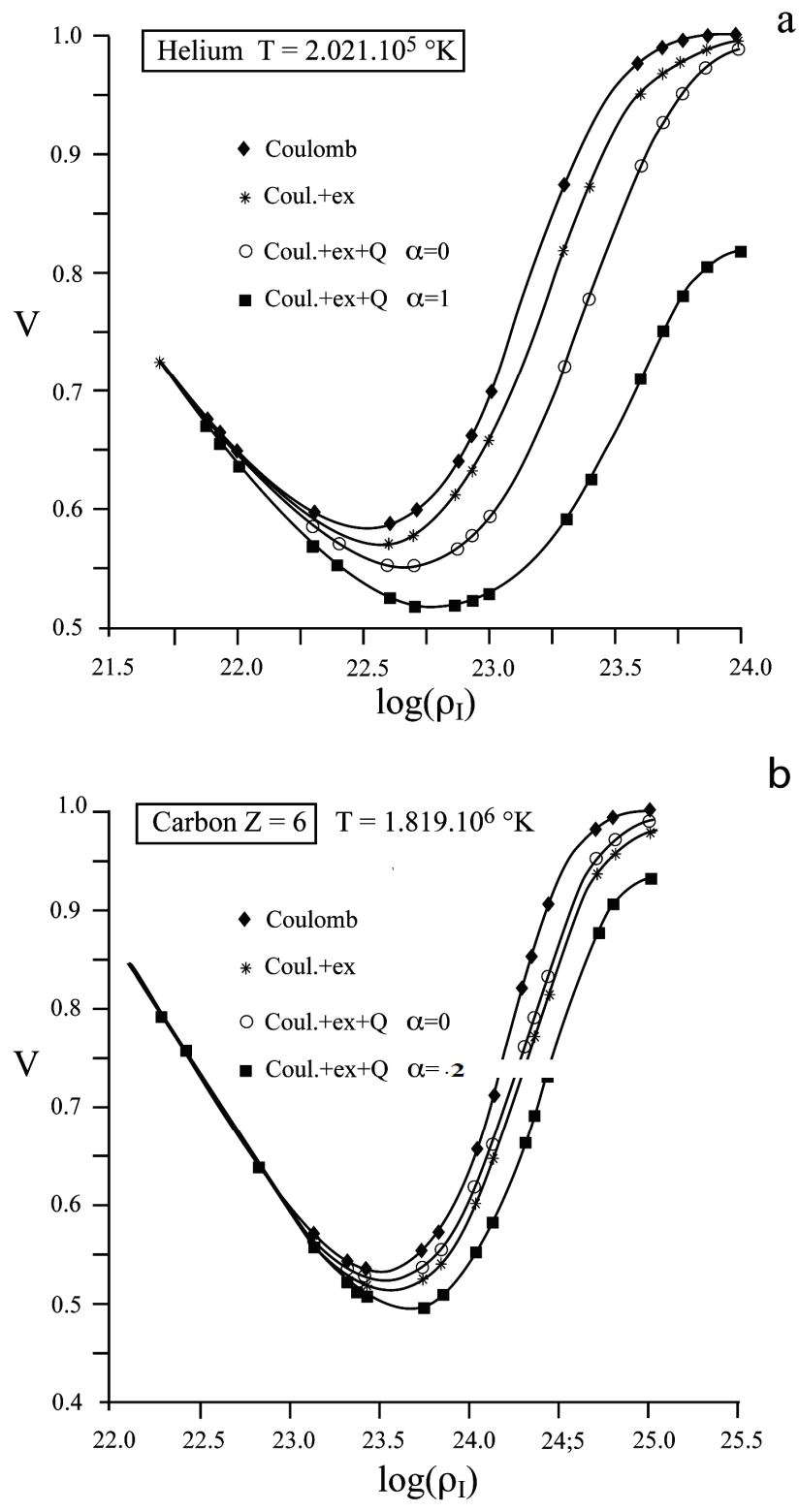

b

Figure 9 - Ionization $v=\rho_{z} / \rho_{\mathrm{I}}$ in a 3 body e-Z-Z $Z_{1}$ plasma with partial sums involvedin Eq.(41) in terms of $\rho_{1}=\rho_{Z}+\rho_{Z_{1}}$.

a) $\mathrm{He}$ at $\mathrm{T}=2.021 \times 10^{5}{ }^{\circ} \mathrm{K}$ for $\mathrm{a}=0$ and $\left.1 ; \mathrm{b}\right) \mathrm{C}$ at $\mathrm{T}=1.819 \times 10^{6} \mathrm{~K}$ for $\mathrm{a}=0$ and 2 


\section{Conclusions}

To summarize, we have demonstrated genuine and He-like effective interactions in dense and hot plasmas analytically derived through explicit wave functions. The novel pseudopotentials have been contrasted to their various $H$-like counterparts $(\alpha=$ 0 ), and thus display highly significant improvements upon the latter. Both approaches converge with increasing atomic number and plasma temperature. Their respective impact on the ionization parameter is also significantly documented, mostly at high density in the pressure dominated regime, which lead us to expect much more accurate results for the equation of state, Hugoniots and transport properties of warm dense matter plasmas of present concern, especially when alpha is larger than one (see Figs. 9). An obvious test of choice for these novel He-like effective interaction could thus be the determination of bremsstrahlung coefficients extending former $\mathrm{H}$ like ones obtained through expression (18) [31].

\section{Acknowledgements}

We gratefully acknowledge pleasant and instructive intercourses about these and related matters with W.Ebeling, D.E. Gericke, W.D. Kraeft, R.M. More, P.Ni, R. Redmer and G. Roepke.

\section{Appendix \\ $a_{b}$ and $a_{c}$ in the Quantum defect method (QDM)[32]}

According to Simons [22], it appears convenient to start from the Fues-like expression for the nonrelativistic hamiltonian for the optical electronhydrogenic core. The hydrogenic core is kept in the $1 \mathrm{~s}$ ground state. The given hamiltonian thus reads as $(\hbar=\mathrm{m}=\mathrm{e}=1)$

$$
H=-\frac{\nabla}{2}-\frac{Z_{1}}{r}+\sum_{\ell=0}^{\infty} P_{\ell} \frac{B(\ell)}{v^{2}}
$$

with projection operator $P_{\ell}=\sum_{m=-\ell}^{m=+\ell}(\ell m((\ell m)$ over subspace pertaining to angular momentum $\ell$, while $\mathrm{B}_{\ell}$ is an adjustable parameter through experimental or theoretical inputs. Pertaining radial equations thus appear under hydrogen like form. Addressing first the bound states set, one has

$$
\left[-\frac{1}{2} d^{2} / d r^{2}+a_{b}\left(a_{b}+1\right) / 2 r^{2}-Z_{1} / r\right] \mu(r)=E \mu(r)(A
$$

has the solutions

$$
\begin{gathered}
E=-Z_{1}^{2} / 2\left(n-1+a_{b}\right)^{2} \\
\mu(r)=C r^{a_{b}+1} \exp (-\varepsilon r) L_{N-l-1}^{2 a+1}(\varepsilon r)
\end{gathered}
$$

with $a_{b}=-\frac{1}{2}+\frac{1}{2}\left[(2 l+1)^{2}+8 B_{b}\right]^{1 / 2}$

$$
\varepsilon=Z /\left(n-1+a_{b}\right)
$$

and where $\mathrm{L}_{\mathrm{p}}^{\mathrm{q}}$ is a generalised Laguerre polynomial. C denotes a normalisation constant while $\mu(\mathrm{r})$ is quadratically integrable provided

$$
\begin{aligned}
& a_{1}>-\frac{3}{2} \\
& \text { et } n-1>1
\end{aligned}
$$

We briefly recall the most significant properties,

(1) The solution of the radial equation (A.2) is analytical.

(2) For a given 1 series, one single parameter $\left(a_{b}\right.$ or $\mathrm{B}_{\mathrm{b}}$ ) determines an orthonormal set of eigen functions and the corresponding spectrum.

(3) The choice of $a_{b}$ is related to the definition of a quantum defect for a series, as can be seen from equation (A.3)

(4) The Fues model appears as a generalisation of the hydrogen case, with the introduction of an effective 'non-integer 1 '.

With increasing $v$, the energies and eigen functions behave 'asymptotically' like those of hydrogen. This extension of the polarization model $\ell \geq 2$ [24] toward larger values yield wave functions without antisymmetrization albeit distinct for total spin $S=0$ or 1 . It is also worthwhile mentioning that $\mathrm{B}_{\mathrm{b}}$ may be given the rather accurate approximant [22]

$$
\mathrm{B}_{\mathrm{b}} \approx-\frac{1}{2} \alpha \mathrm{e}^{2} \frac{\left\langle\mathrm{r}^{-4}\right\rangle_{\mathrm{H}}}{\left\langle\mathrm{r}^{-2}\right\rangle_{\mathrm{H}}}
$$


in terms of the $Z_{1}$ core polarizability in $10^{-24} \mathrm{~cm}^{2}$ and hydrogenic radial averages $\left\langle\mathrm{r}^{-\mathrm{p}}\right\rangle_{\mathrm{H}}$ with $\mathrm{p}$ integer $>0$. Similarly, corresponding positive energy continuum states show up as eigenstates of Eq. (A.1) with a $B_{c}$ parameter featuring $a_{c}$ through $\ell(\ell+1)+2 \mathrm{~B}_{\mathrm{c}}=\mathrm{a}_{\mathrm{c}}\left(\mathrm{a}_{\mathrm{c}}+1\right) \mathrm{a}_{\mathrm{b}}$ and $\mathrm{a}_{\mathrm{c}}$ are related by the Levinson theorem near the ionization edge with

$$
\mathrm{a}_{\mathrm{b}}(\mathrm{n} \rightarrow \infty, \ell)=\mathrm{a}_{\mathrm{c}}(\mathrm{k}=0, \ell)
$$

Present QDM phase shifts w.r.t. Coulombic ones, are then givent as $\left(\eta=Z_{1} / k\right)[21]$

$$
\begin{gathered}
\delta_{\ell}\left(k^{2}\right)=\arg \Gamma\left(1+a_{c}-i \eta\right)- \\
-\arg \Gamma(1+\ell-i \eta)+\frac{\pi}{2}\left(\ell-a_{c}\right)
\end{gathered}
$$

in terms of $\mathrm{a}_{\mathrm{c}} \cdot \delta_{\ell}\left(\mathrm{k}^{2}\right)$ and $\mathrm{a}_{\mathrm{c}}$ are both dependent on $\mathrm{Z}$ and $\mathrm{S}$.

\section{References}

1. A.V. Filinov et al. Temperature-dependent quantum pair potentials and their application to dense partially ionized hydrogen plasmas // Phys. Rev. E. - 2004. - Vol. 70. - P. 046411

2. A.V. Filinov et al. Improved Kelbg potential for correlated Coulomb systems // J. Phys. A. - 2003. - Vol. 36. P. 5957.

3. G. Kelbg. On the transport properties of a dense fully-ionized hydrogen plasma// Ann. Phys. Leipzig. - 1963. Vol. 12. - P. 219

4. C. Deutsch. Nodal expansion in a real matter plasma // Phys. Lett. A. - 1977. - Vol. 60. - P. 317

5. C. Deutsch, Y. Furutani, M.M Gombert. Nodal expansions for strongly coupled classical plasmas // Phys. Rep. 1981. - Vol. 69. - P. 85

6. H. Minoo, M.M. Gombert, C. Deutsch. Temperature-dependent Coulomb interactions in hydrogenic systems // Phys. Rev.A. - 1981. - Vol. 23. - P. 924.

7. M.M. Gombert. Temperature-dependent pseudopotential between two pointlike electrical charges // Phys. Rev. E. - 2002. - Vol. 66. - P. 066407.

8. M.M. Gombert, Temperature dependent pseudopotential between two pointlike electrical charges // Contrib. Plasma Phys. - 2003. - Vol. 43. - P. 302.

9. H. Wagenknecht, W.E. Ebeling, A. Förster. Effective potentials, energies, and pair distribution functions of plasmas by Monte - Carlo simulations // Contrib. Plasma Phys. - 2001. - Vol. 41. - P. 15.

10. A. E.S. Green, D.L. Sellin, A.S. Zachor. Analytic independent-particle model for atoms // Phys. Rev. - 1969. Vol. 184. - P.1.

11. D.A. Chapman, D.O. Gericke. Analysis of Thomson scattering from nonequilibrium plasmas // Phys. Rev. Lett. - 2011. - Vol. 107. - P. 165004.

12. D.O. Gericke et al. Structural properties of warm dense matter // J.Phys: Conf. Series. - 2010. - Vol. 220. - P. 012001.

13. J. Hasegawa, Y. Nakajima, K.Sakai, M.Yoshida, S.Fukata, K.Nishigori, M.Kojima, Y.Oguri, M.Nakajima, K.Horioka, M.Ogawa, U.Neuner. Energy loss of $6 \mathrm{MeV} / \mathrm{u}$ iron ions in partially ionized helium plasma // Nucl. Instrum. Meth. A. - 2001. - Vol. 464. - P. 165004.

14. H. Reinholz. Dielectric and optical properties of dense plasmas // Ann. Phys-Paris - 2005. - Vol. 30. - P.1.

15. M.Lisal, W.R.Smith, I.Nezbeda. Molecular simulation of multicomponent reaction and phase equilibria in MTBE ternary system // J. Chem. Phys. - 2000. - Vol. 113. - P. 4885.

16. B.Militzer. Path integral Monte Carlo and density functional molecular dynamics simulations of hot, dense helium // Phys. Rev.B. - 2009. - Vol. 79. - P. 155104.

17. T.S.Ramazanov, K.N. Dzhumagulova, M.T.Gabdullin. Effective potentials for ion-ion and charge-atom interactions of dense semiclassical plasma // Phys.Plasmas - 2010. - Vol. 17. - P. 042703

18. J.Dai, Y.Hoo and J.Yuan. Nonequilibrium Fluctuation Relations in a Quantum Coherent Conductor// Phys. Rev.Lett. - 2010. - Vol. 104. - P. 254001

19. E.L. Pollock. Properties and computation of the Coulomb pair density matrix // Comput. Phys.Commun. 1988. - Vol. 52. - P. 49.

20. G.W.F. Drake, R.A. Swainson. Quantum defects and the 1/n dependence of Rydberg energies: Second-order polarization effects // Phys. Rev. A. - 1991. - Vol. 44. - P. 5448.

21. A. Valance et al., Theoretical determination of van der Waals $\mathrm{Ne}(2 \mathrm{p} 61 \mathrm{~S} 0) \mathrm{He}(21,3 \mathrm{~S} 0)$ potentials // J. Phys.B. 1986. - Vol. 19. - P. 3919.

22. G. Simons. New model potential for pseudopotential calculations // J. Chem Phys. - 1971. - Vol. 55. - P. 756. 
23. E. Clementi, C. Roetti. Roothaan-Hartree-Fock atomic wavefunctions: basis functions and their coefficients for ground and certain excited states of neutral and ionized atoms, $\mathrm{Z}<=54$ // Atomic Data and Nucl. Data Tables. - 1974. Vol. 14. - P. 177.

24. H.A. Bethe, E.E. Salpeter. Quantum Mechanics of one-and two-electron atoms //A Plenum/Rosetta Edition. NY. - 1977, - P. 15.

25. C. Deutsch. Polarization model for the excited states of neutral helium// Phys. Rev. A. - 1971. - Vol. 2. - P. 43.

26. C. Deutsch. Rydberg states of HeI using the polarization model // - 1976. - Vol. 13. - P. 2311.

27. H. Rahal, Dr. Sc. Thesis // Univ. Paris-Sud, 1995 (unpublished)

28. H.A.Bethe, E.E.Salpeter. Quantum Mechanics of one-and two-electron atoms // A Plenum/Rosetta Edition,NY. - 1977, - P. 153.

29. H. Rahal, M.M. Gombert. Theoretical derivation of bound and continuum states of two-electron ions with model potential // J. Phys. B. - 1997. - Vol. 21. - P. 4695.

30. C. Deutsch, M.M. Gombert and H. Minoo. Classical modelization of symmetry effects in the dense hightemperature electron gas // Phys. Lett. A. - 1978. - Vol. 66. - P. 381.

31. H. Furukawa, K. Nishihara. Fermi-degeneracy and discrete-ion effects in the spherical-cell model and electronelectron correlation effects in hot dense plasmas // Phys.Rev. A. - 1992. - Vol. 46. - P. 3532.

32. M.J. Seaton. Quantum defect theory // Rep. Prog. Phys. - 1983. - Vol. 46. - P. 167. 